\title{
Analysis of Stereoscopic Images as a New Method for Daylighting Studies
}

\author{
CLAUDIA MOSCOSO, BARBARA MATUSIAK, U. PETER SVENSSON, and KRZYSZTOF ORLEANSKI, \\ Norwegian University of Science and Technology
}

\begin{abstract}
This article presents the comparison analysis and results of an experiment designed with two presentation modes: real environments and stereoscopic images. The aim of this article is of a methodological nature, with a main objective of analyzing the usability of stereoscopic image presentation as a research tool to evaluate the daylight impact on the perceived architectural quality of small rooms. Twenty-six participants evaluated 12 different stimuli, divided in equal parts between real rooms and stereoscopic images. The stimuli were two similar rooms of different achromatic-colored surfaces (white and black) with three different daylight openings in each room. The participants assessed nine architectural quality attributes on a semantic differential scale. A pragmatic statistical approach (Bland-Altman Approach) for assessing agreement between two methods was used. Results suggest that stereoscopic image presentation is an accurate method to be used when evaluating all nine attributes in the white room and nearly all attributes in the black room.
\end{abstract}

Categories and Subject Descriptors: I.4.0 [Image Processing and Computer Vision]: General—Image displays; I.4.8 [Image Processing and Computer Vision]: Scene Analysis—Stereo; I.6.3 [Simulation and Modeling]: Applications; I.6.8 [Simulation and Modeling]: Types of Simulation-Visual; J.5 [Arts and Humanities]: Architecture

General Terms: Design, Experimentation, Human Factors, Measurement, Reliability

Additional Key Words and Phrases: Daylighting studies, achromatic color, stereoscopic imaging, method comparison, aesthetic perception, visualization

ACM Reference Format:

Claudia Moscoso, Barbara Matusiak, U. Peter Svensson, and Krzysztof Orleanski. 2015. Analysis of stereoscopic images as a new method for daylighting studies. ACM Trans. Appl. Percept. 11, 4, Article 21 (January 2015), 13 pages.

DOI: http://dx.doi.org/10.1145/2665078

\section{INTRODUCTION}

The difficulty of conducting full-scale experimental studies has been stated by numerous researchers [Newsham et al. 2005; Villa and Labayrade 2013]. To conduct rigorous scientific architectural or lighting research in Real Environments (REs) brings a series of challenges even for the most experienced

This study was carried out as a part of a $\mathrm{PhD}$ research project funded by the Norwegian University of Science and Technology. Authors' addresses: C. Moscoso and B. Matusiak, Department of Architectural Design, Form and Colour Studies, Norwegian University of Science and Technology, Alfred Getz vei 3, NO-7491, Trondheim, Norway; emails: \{claudia.moscoso, barbara. matusiak\}@ntnu.no; U. P. Svensson and K. Orleanski, Department of Electronics and Telecommunications, Norwegian University of Science and Technology, O.S. Bragstads plass 2, NO-7491, Trondheim, Norway; emails: \{Svensson, krzysztof.orleanski\}@ iet.ntnu.no.

Permission to make digital or hard copies of all or part of this work for personal or classroom use is granted without fee provided that copies are not made or distributed for profit or commercial advantage and that copies bear this notice and the full citation on the first page. Copyrights for components of this work owned by others than the author(s) must be honored. Abstracting with credit is permitted. To copy otherwise, or republish, to post on servers or to redistribute to lists, requires prior specific permission and/or a fee. Request permissions from permissions@acm.org.

2015 Copyright is held by the owner/author(s). Publication rights licensed to ACM.

ACM 1544-3558/2015/01-ART21 \$15.00

DOI: http://dx.doi.org/10.1145/2665078 
researcher. For example, Sprow et al. [2009] discuss the complexity and difficulty of testing a large number of participants in a laboratory setting for obtaining more robust statistical results. In another example, Pellegrino [1999] had to stretch out her experimental hours late into the evening until daylight could be excluded from her electrical lighting settings. She discussed that this may have been an influential factor in her collected data.

In order to perform varied architectural and environmental research within the daylighting field without encountering similar problems, static imaging (e.g., photographs, slides, and computerrendered images) is probably one of the most-used simulation methods in experimental research. For example, Newsham et al. [2002] found similar results between the scores that participants gave to grayscale images and those from real settings. Mahdavi and Eissa [2002] concluded that the results of the evaluation of non-daylit office spaces in REs were similar to the results obtained by using highquality computer renderings. Villa and Labayrade [2013] compared the results obtained by an online laboratory experiment to evaluate virtual luminous environments to those obtained in a real laboratory experiment. Their findings revealed no differences between the data collected in the laboratory and online.

Stamps [1990] claims that since a considerable amount of environmental research uses photographic representation, there is a need to find out how well photographs simulate environments. This is an important task because a new confidence in screened or projected static imaging has produced lighting research using only this method to gather data [Newsham et al. 2005; Naoyuki 2005].

Nonetheless, static images may be considered an incomplete representation of the RE. For example, Matusiak and Sudbø [2008] used static images produced with the lighting simulation program Radiance. Their findings concluded that the impression of width and height were perceived rather equally in the real and in the simulated space, yet the question about the size impression of the room could not be answered by computer simulations alone. An environmental simulation will always act as a reduced environment in which not all the senses can be stimulated exactly as in real spaces.

However, in order to appraise an indoor environment, one of the most important senses is vision. Our visual perception seems to have a larger capacity for recognition and information processing than any of the other senses. The challenge lies, then, in producing imaging with enough experiential realism (i.e., the room is experienced as having a real existence). This concept has been discussed by de Kort et al. [2003]: If a display can be more perceptually realistic, then the participants' scores to stimuli in a simulated environment can be similar to those in an RE. To increase experiential realism, a third dimension to static imaging can give a sense of presence (i.e., the state of the observer actually "being there") in the environment.

Stereoscopic imaging (SI) has been sparsely used in lighting research. Wienold et al. [1998] used stationary virtual reality to study a prediction method of user acceptance of daylighting systems. Fontoynont et al. [2007] also used SIs to find a correlation between lighting quality descriptors and the semantic characterization of luminous scenes. However, very few research efforts are encountered that use SI as the only tool for investigating the impact of daylighting on the visual appearance of a room.

The validity of SI as an architectural research tool has not been proved yet. In order to investigate SI as a tool for daylighting and architectural research, SI has to be evaluated together with an RE. A research question then arises: Can an SI presentation give results that are similar to real room experiments when studying daylighting in architecture? Comparisons between REs and simulated environments seem to be focused on different areas of expertise, including depth perception [Akai 2007], color appearance [Billger et al. 2004], size perception [Geuss et al. 2012], distance estimation [Ziemer et al. 2009], and shape evaluation [Okubo 2011]. However, little research activity is encountered that compares assessments of daylight in RE versus simulated rooms using SIs in architectural research. 
Preliminary results of a pilot study [Moscoso and Matusiak 2013] show that SI can serve as a tool for studying limited attributes of a room under certain luminous conditions. If the results obtained under SI are not significantly different from the results obtained under an RE, then the validity of SI results may be trusted. High-definition SI may offer a better approximation to reality than twodimensional static imaging. In addition, it may be possible to retain control over the different stimuli and variables of an experiment. Other advantages of SI as a research tool range from the reduction of cost for experimental setting construction to the possibility of reaching bigger audiences and obtaining more robust statistical results.

\section{METHOD AND PROCEDURE}

Two real temporary rooms, one black and one white, were constructed at the Room Laboratory (Roomlab) located at the Norwegian University of Science and Technology (NTNU), Trondheim, Norway. The SIs were taken directly from the real rooms and shown on a silver screen in the same laboratory. The study was carried out in three different experimental sessions: two sessions with eight participants and one session with 10 .

\subsection{Participants}

The participants were recruited via email, visual advertisements on the NTNU campus, and through the university's intranet site. Participation was voluntary and was rewarded with a free movie ticket to see a movie of choice. The total sample consisted of 26 participants (14 male, 12 female), aged between 24 and 62 years $(M=32.7$ years, $S D=8.4$ years), with and without architecture and/or lighting training. Participants were from different nationalities and cultural backgrounds. After approval from the Norwegian Social Science Data Services (NSD; Norsk samfunnsvitenskapelig datatjeneste), all participants were tested for visual acuity prior to the experiment to ensure that they did not have particular vision impairments that could compromise the collected data. The two vision tests conducted were (i) the Luminance Contrast Test (Spatial Contrast Sensitivity; Valberg [2005]) via the computer software $\operatorname{VigraC}^{\circledR}$ and using the Michelson Plot curve to test five different spatial frequencies and (ii) the Stereoscopic Vision Test, making use of the Random Dot 2 Stereo Acuity Test. The results of the vision tests were only given to each participant after testing and were not reported in any way. Every participant read a "General Information Sheet for Participants" containing a full explanation of the experimental session. After having read the information sheet and heard the oral instructions, each participant signed an individual form in which he or she freely consented to participate in the study.

\subsection{Experimental Setting: Stimuli}

2.2.1 Real Environment Characteristics. Two rooms of equal dimensions $(3.00 \mathrm{~m} \times 3.60 \mathrm{~m})$ with similar openings and furniture configuration were constructed. One room had white room (WR) surfaces (ceiling, walls, and floor), and the other had black room (BR) surfaces (ceiling, walls, and floor). For each room color, three different daylight openings were assigned. In this way, each room was constructed only once, but the size of the window was changed between stimuli presentation. The windows were small $(1 \mathrm{~m} \times 1 \mathrm{~m})$, medium $(2 \mathrm{~m} \times 1 \mathrm{~m})$, and large $(2 \mathrm{~m} \times 1.5 \mathrm{~m})$. These rooms were built with the aid of "wall bricks" (boxes of $50 \mathrm{~cm} \times 50 \mathrm{~cm} \times 25 \mathrm{~cm}$ ) and preconstructed wall panels $(60 \mathrm{~cm} \times 250 \mathrm{~cm} \times 5 \mathrm{~cm}$ ). To build windows, metal frames of equal dimensions to the "bricks" were used. The furniture had an abstract character and was created using the same "wall bricks." In addition, a chair, lamp, desk plate, and bedding set was set in place to create a strong association with a "student room." In both rooms, the furniture was situated in the same coordinates relative to the position of the entrance and the window (see Figure 1). 


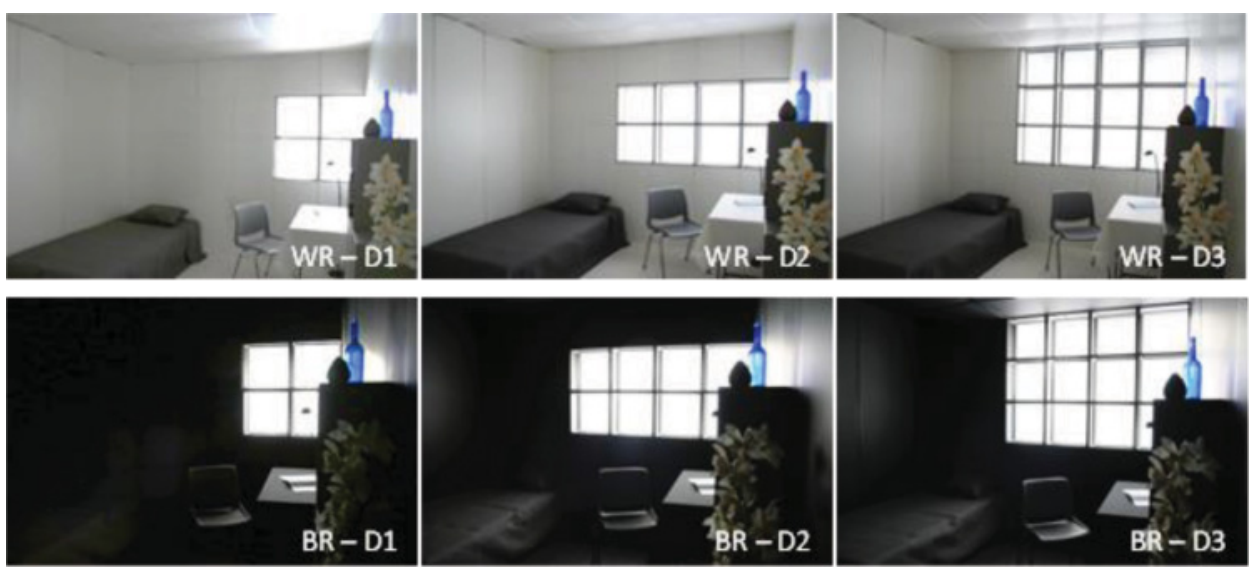

Fig. 1. Experimental setting - real environments (c)Claudia Moscoso).

Table la. Mean Illuminance Values Measured at Two Different Points in Each Room

\begin{tabular}{|l|l|r|r|c|}
\hline Room & Window $(\mathrm{M})$ & Bed $(\mathrm{lx})$ & Desk $(\mathrm{lx})$ & Mean $(\mathrm{lx})$ \\
\hline Black & Small $(1 \times 1)$ & 1.5 & 87.3 & 44.4 \\
& Medium $(2 \times 1)$ & 20.0 & 110.8 & 65.4 \\
& Large $(2 \times 1.5)$ & 31.8 & 162.3 & 97.1 \\
\hline \multirow{5}{*}{ White } & Small $(1 \times 1)$ & 65.2 & 220.5 & 142.8 \\
& Medium $(2 \times 1)$ & 138.4 & 328.5 & 233.4 \\
& Large $(2 \times 1.5)$ & 227.1 & 497.5 & 362.3 \\
\hline
\end{tabular}

Both rooms had abstract desks of the same color as the wall surfaces (a white desk in the white room, a black desk in the black room) and the rest of the furniture (i.e., chair, bed, and bookshelf) were of a gray color in both rooms, in order to maintain achromatic colors. The nominal color of the wall elements and main furniture was registered with the Natural Color System (NCS). For the black room, the lateral walls presented a nominal color of S8500-N (Reflectance factor $\mathrm{Y} 1=6$ ) and the window walls a nominal color of S9000-N (Reflectance factor $\mathrm{Y} 1=4$ ). The white room had a nominal color of S0502-Y (Reflectance factor Y1 = 87) for the lateral walls and a nominal color of S0500-N (Reflectance factor Y1 = 87) for the window walls. The reflectance factor Yl given by NCS is the CIE tristimulus value $\mathrm{Y}$, measured with an integrating sphere type photospectrometer with an included specular component. This measurement method prevents differences due to the influence of the gloss level of the measured surface (diffuse or glossy surfaces) [SIS 1998].

To have better control over the illuminance fluctuations that are typical for natural light and to avoid an outside view, white translucent curtains (transmittance 46\%) were used. The position of the rooms inside the Roomlab was chosen such that the "windows" in the white and black rooms were oriented to the northeast. This orientation ensured no direct sunlight after midday. Additionally, the experiment was carried out during periods with overcast sky conditions. The illuminance values were measured using a BEHA 93408 digital lux meter (Table Ia). The luminance values (Table Ib) and luminance pictures (see Figure 2) were taken with an EOS350D digital reflex camera and analyzed with the software LMK 2000. The participants were able to choose their place in the room, but they were instructed to do their evaluation while facing the window wall. 
Table Ib. Luminance Values $\left(\mathrm{u}=\mathrm{cd} / \mathrm{m}^{2}\right)$ Measured at Six Points in Each Stimulus

\begin{tabular}{|c|c|r|r|c|}
\hline \multicolumn{2}{|l|}{ Room } & \multicolumn{1}{|c|}{ D1 } & \multicolumn{1}{c|}{ D2 } & D3 \\
\hline Black & Min & 13.21 & 4.22 & 159.37 \\
& Max & 37.20 & 184.22 & 203.07 \\
& Mean & 34.15 & 145.84 & 238.94 \\
\hline White & Min & 187.24 & 266.16 & 302.47 \\
& Max & 221.98 & 305.77 & 357.09 \\
& Mean & 292.88 & 387.94 & 460.35 \\
\hline
\end{tabular}
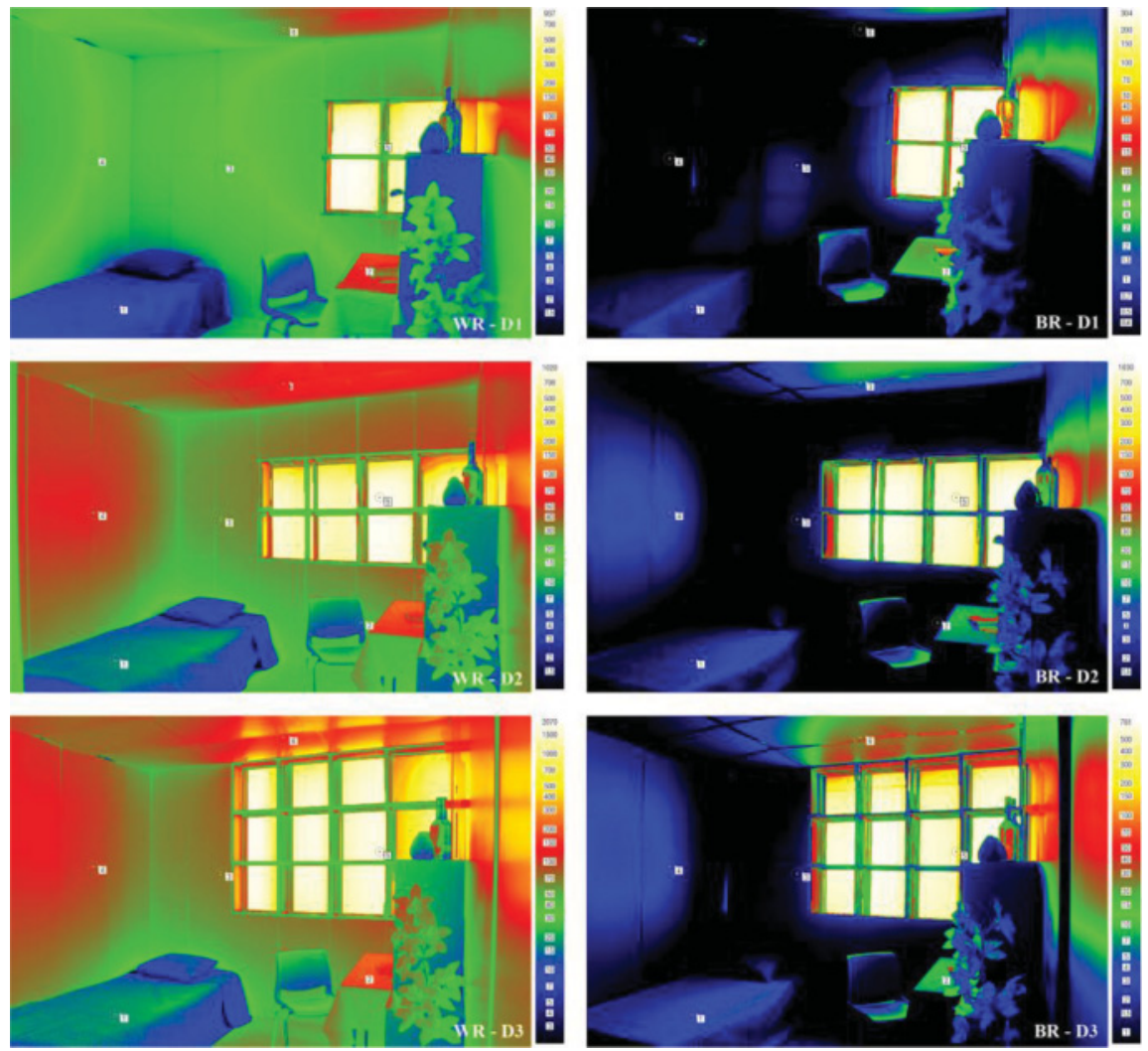

Fig. 2. Luminance pictures measured at six different points in each RE and analyzed on the LMK2000. All values were measured each time before the participants evaluated the rooms (c)Claudia Moscoso).

2.2.2 Stereoscopic Image Characteristics. The participants also evaluated three-dimensional (3D) pictures taken with two conventional photographic cameras (OLYMPUS SP-800UZ). The cameras were used with parallel optical axes and placed at an observer's vision height $(1.65 \mathrm{~m})$. They were mounted horizontally on a tripod with a distance of $10.5 \mathrm{~cm}$ between the middle points of the lenses (due to the size of the cameras) when taking the pictures. One camera captured a "left eye view," and the second camera, positioned right beside of the first camera, captured the "right eye view." All photography settings were equal in both cameras when taking all pictures, having ISO, aperture, shutter speed, and white balance in automatic (adaptive) mode. This was done under the premise that our visual 


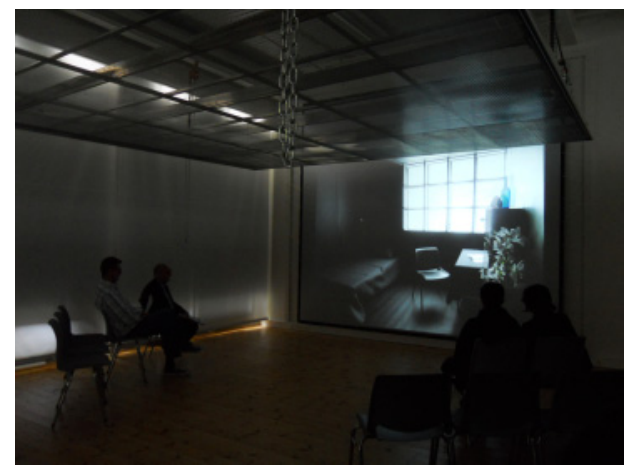

Fig. 3. Experimental setting: stereoscopic images evaluations (c)Claudia Moscoso).

system also adapts. Two sets of SIs were taken in each room, both directed toward the window wall. The SIs were taken under overcast sky conditions to match the type of sky conditions during the experimental session. The first SI was taken from the entrance, and the second SI was taken from the left-side corner opposite to the entrance (see Figure 3). The images received no special editing treatment, except in the case of the images from the black room with the small window where the brightness was computationally increased by $80 \%$ to match the perceived brightness from the RE.

The images were displayed using commercial SI player software. They were then projected and superimposed with two Full High-Definition OPTOMA Projectors EP1080 of more than 5,000 ANSI lumen, with a resolution of 1,400 pixels $\times 1,050$ pixels each through different circular polarized filters. To eliminate the possible loss of light due to polarization, the SIs were projected on a silver screen known to have an especially good performance with 3D imaging. The luminance of each projected SI was measured using a Minolta LS-100 luminance meter. Measurements showed that the SI with the lowest mean luminance was the black room with small window (i.e., $10 \mathrm{~cd} / \mathrm{m}^{2}$ ). Under these luminance conditions, participants evaluated the SIs against photopic vision. Participants made use of circular polarized glasses such that each eye received a unique image and the limitations of head movement were avoided. The participants were seated at a distance no larger than $4.0 \mathrm{~m}$ from the silver screen (see Figure 3). The viewing configuration was as if the participant was moving in the room from side to side looking in the direction of the window wall. This viewing configuration was selected to emulate the instructed viewing evaluation of the REs (as described in Section 2.2.1) and to facilitate comparison. The participants were free to choose if they wanted to be seated or standing on either side. During the assessments, the participants' positions were carefully observed by the experimenter.

\subsection{Dependent Variables}

Environmental research has identified six main types of aesthetic attributes: order, complexity, openness, naturalness (i.e., the presence of vegetation, water, or mountains), upkeep, and historical significance [Nasar 2000]. Since the present research directs attention to the perceived quality of an interior space, naturalness and upkeep (civilities) are excluded from the study. These two attributes can relate to both interior and exterior architecture, but they are not as strongly related to interior spaces as are the others. Needless to say, historical significance does not apply in the present study, considering that it refers only to new environments. Kaplan and Kaplan [1989] discuss the importance of spaciousness and spatial definition in people's perception of the built environment, and therefore these attributes have been included in this study. Other aesthetic attributes were selected from a group of scales commonly used in architecture and lighting research. Due to the focus of the experiment on 
studying aesthetic attributes, cognitive judgments (e.g., brightness) were not selected to be studied. Participants evaluated the REs and SIs using a seven-step semantic differential scale [Osgood et al. 1971]. To avoid bias, participants were not given a numerical rating scale to use when evaluating the view. However, a 1-7 value scale was assigned when the data were analyzed (e.g., $1=$ Dull to $7=$ Exciting). The evaluated attributes (dependent variables) were: Unpleasant-Pleasant (e.g., the room was perceived as agreeable or enjoyable), Dull-Exciting (e.g., the room provoked an enjoyable and active state of mind in the observer), Chaotic-Ordered (e.g., the room was arranged suitably as a student's study room), Simple-Complex (e.g., the room was perceived as having a complex visual richness in its parts), Illegible-Legible (e.g., the observer could clearly see all the room details), IncoherentCoherent (e.g., the room was considered consistent with its function as a student's study room in relation to the amount of daylight), Tight-Spacious (e.g., the room was perceived as spacious), ClosedOpen (e.g., the room was perceived as having enough openings), and Spatially Undefined-Spatially Defined (e.g., it was not difficult to see the room's boundaries). The dictionary definitions of each attribute and examples of their meaning (such as those given here) were both written in the participants questionnaires and orally explained to them at the oral task introduction prior to the experimental session. Participants were asked to place only one mark on the scale according to their own evaluation. The nine scales were printed in groups on three sheets of paper, which the participants were requested to fill out before moving on to the next stimulus.

\subsection{Experimental Procedure}

All three experimental sessions were carried out at midday, when the daylight level is at its highest. Each session had an approximate duration of 2.5 hours. The protocol was maintained equally in all sessions. Each day, participants were welcomed to the Roomlab at NTNU, where they received practical and task information, as well as the verbal and written instructions for the experiment. Participants were then asked to sign a consent form. The group of participants was randomly divided into three subgroups. The first group started evaluating the RE-White Room, the second group started evaluating the RE-Black Room, and the third group started with the evaluation of the SI. The groups were rotating among the different stimuli presented. In this way, all the subgroups could evaluate every single stimulus (three different window sizes: small window (D1), medium window (D2), and large window (D3) in each RE; WR and BR; and the same number of stimuli in the SIs). By randomizing stimuli presentation, the bias connected to the context effect as well as sequential contraction bias was controlled. All spaces were supervised by the experimenter to ensure quality in the presentation of the stimuli, and illuminance and luminance measurements were taken prior to participants' evaluations.

Participants were free to move about in the room, but they were instructed in which direction to look, which was toward the window wall. The participants filled out one written questionnaire for each presented stimulus. In total, there were 12 different stimuli (six in RE and six in SI). There was no predetermined time for participants to make their evaluations; they were able to take as much time as needed to evaluate both REs and the SIs. During the interstimulus intervals (change of stimulus, window size in the REs), participants were served snacks and drinks and had time to relax. These experimental pauses and the randomization of the stimuli presentation were planned to avoid fatigue during SI observation and throughout the experimental session. Additionally, no signs of fatigue or discomfort were observed nor reported by the participants. The participants were instructed not to comment on their experiment experiences among themselves. For the SI evaluation, a set of two SIs was presented. The first SI was displayed for 30 seconds, followed by the second SI. As described in Section 2.2.2, these two sets of SI belonged to each room but were taken from different angles. Provided that these two SI sets corresponded to the same room, participants were asked to make their evaluations after observing both sets. 
At the end of the quantitative part of the experiment, participants who were voluntarily willing to participate in a qualitative interview stayed in the Roomlab. The interviews were private, and the main experimenter sat alone with each participant for a debriefing time and to gather extra information.

\section{RESULTS}

\subsection{Analysis Strategy}

The objective of the presented study is to analyze, using observers' ratings, if SI presentation is a method that can substitute for real room experiments in the quality evaluation of luminous environments. The answer to this question can be obtained by a method-comparison study. Statistical tests, such as correlation analysis and linear regression, have been discussed as misleading techniques in method comparison studies [Altman and Bland 1983]. Certainly, performed correlation tests gave insufficient results for this study. The Pearson's product-moment correlation coefficient was computed first to assess the relationship between the mean ratings obtained from the WR-RE and WR-SI. There was a very strong positive correlation between the two variables, $r=0.879, n=27, p=0.000$. The relationship between the mean ratings from the BR-RE and BR-SI also showed a very strong positive correlation between the variables, $r=0.827, n=27, p=0.000$. These results were a good indication of the strength of the relation between RE and SI. However, this correlation does not show the level of agreement between the two studied methods.

Within medical research, a methods-comparison approach is used based on graphical techniques and simple calculations. This approach, identified as the Limits of Agreement (LoA) method or BlandAltman approach was chosen to be applied to the reported study [Bland and Altman 1986]. The BlandAltman approach recognizes that it is very unlikely that two methods will agree exactly by giving equal ratings for each experiment participant. The objective is to know how large the difference is between the ratings of the established method and the tested method. By calculating the mean difference (i.e., accuracy) of the two methods, it becomes possible to obtain the systematic difference between the ratings.

The data were divided into two groups considering the variable "room color" (i.e., first group: WR; second group: BR). Thus, the data could be analyzed using replicated measurements. Since the evaluations of each room were made under three different conditions (i.e., WR with three different window sizes and BR with three different window sizes), the selected Bland-Altman approach was the method with multiple observations per participant where the true value varies [Bland and Altman 2007]. Hence, the variable "room color" was compared between the two levels (white and black), and the variable "window size" was treated as 78 different observations (26 participants evaluated 3 different window sizes in each room), according to the Bland-Altman approach. Both rooms were evaluated separately on all nine assessed attributes. In this way, the study reports 18 separate method-comparison analyses.

Considering the seven steps of the semantic differential scale, the a priori criterion for the mean difference considered acceptable by the authors is $<1$ unit. The precision, estimated by the LoA, is given as part of the results.

\subsection{Bland-Altman Method with Multiple Observations (True Value Varies)}

Numerical results are presented in Table II. For brevity and clarity and due to the nature of the experimental design (number of stimuli and dependent variables), we do not show the complete set of graphical representations for each stimulus and attribute in this article, but present only two as examples. However, the 18 graphical plots are available from the authors upon request. 
Table II. Summarized Numerical Results from the Bland-Altman Method for Each Attribute in the White (WR) and Black Room (BR)

\begin{tabular}{|c|c|c|c|c|c|c|c|c|c|c|c|}
\hline & & $\begin{array}{c}\text { Mean } \\
\text { Difference }\end{array}$ & (Mear & $\begin{array}{l}\text { I } \\
\text { rence) }\end{array}$ & SD & $\begin{array}{c}\text { Lower } \\
\text { LoA }\end{array}$ & & & $\begin{array}{l}\text { Upper } \\
\text { LoA }\end{array}$ & & \\
\hline \multirow{2}{*}{ Pleasantness } & WR & 0.4 & 0.04 & 0.81 & 1.71 & -2.92 & -3.58 & -2.25 & 3.76 & 3.10 & 4.43 \\
\hline & $\mathrm{BR}$ & 0.4 & -0.05 & 0.90 & 2.09 & -3.68 & -4.50 & -2.86 & 4.53 & 3.71 & 5.35 \\
\hline \multirow{2}{*}{ Excitement } & WR & 0.4 & 0.12 & 0.78 & 1.45 & -2.40 & -2.97 & -1.83 & 3.30 & 2.73 & 3.86 \\
\hline & $\mathrm{BR}$ & 0.2 & -0.24 & 0.65 & 1.98 & -3.68 & -4.45 & -2.91 & 4.09 & 3.32 & 4.86 \\
\hline \multirow{2}{*}{ Order } & WR & -0.3 & -0.55 & -0.12 & 0.96 & -2.22 & -2.59 & -1.84 & 1.55 & 1.18 & 1.93 \\
\hline & $\mathrm{BR}$ & -0.2 & -0.52 & 0.14 & 1.47 & -3.08 & -3.65 & -2.50 & 2.69 & 2.12 & 3.27 \\
\hline \multirow{2}{*}{ Complexity } & WR & 0.0 & -0.32 & 0.29 & 1.36 & -2.67 & -3.20 & -2.14 & 2.65 & 2.12 & 3.18 \\
\hline & $\mathrm{BR}$ & -0.1 & -0.63 & 0.34 & 2.15 & -4.35 & -5.19 & -3.52 & 4.07 & 3.23 & 4.91 \\
\hline \multirow{2}{*}{ Legibility } & WR & 0.2 & -0.05 & 0.43 & 1.06 & -1.88 & -2.29 & -1.46 & 2.26 & 1.85 & 2.67 \\
\hline & $\mathrm{BR}$ & 0.4 & -0.04 & 0.91 & 2.10 & -3.68 & -4.50 & -2.86 & 4.55 & 3.73 & 5.37 \\
\hline \multirow{2}{*}{ Coherence } & WR & 0.1 & -0.19 & 0.40 & 1.31 & -2.47 & -2.98 & -1.96 & 2.67 & 2.16 & 3.18 \\
\hline & $\mathrm{BR}$ & -0.4 & -0.85 & 0.01 & 1.90 & -4.15 & -4.90 & -3.41 & 3.31 & 2.56 & 4.05 \\
\hline \multirow{2}{*}{ Spaciousness } & WR & 0.6 & 0.28 & 0.92 & 1.43 & -2.20 & -2.76 & -1.64 & 3.40 & 2.85 & 3.96 \\
\hline & $\mathrm{BR}$ & 0.7 & 0.32 & 1.09 & 1.69 & -2.60 & -3.26 & -1.94 & 4.01 & 3.35 & 4.67 \\
\hline \multirow{2}{*}{ Openness } & WR & 0.6 & 0.26 & 0.97 & 1.58 & -2.49 & -3.10 & -1.87 & 3.72 & 3.10 & 4.33 \\
\hline & $\mathrm{BR}$ & 0.3 & -0.21 & 0.72 & 2.07 & -3.80 & -4.61 & -2.99 & 4.31 & 3.51 & 5.12 \\
\hline \multirow{2}{*}{$\begin{array}{l}\text { Spatial } \\
\text { Definition }\end{array}$} & WR & 0.0 & -0.22 & 0.29 & 1.13 & -2.18 & -2.63 & -1.74 & 2.26 & 1.82 & 2.70 \\
\hline & BR & 0.8 & 0.33 & 1.23 & 2.00 & -3.14 & -3.92 & -2.36 & 4.70 & 3.92 & 5.48 \\
\hline
\end{tabular}

*95\%-95\% Confidence Interval (CI); SD, Standard Deviation; L-LoA, Lower Limit of Agreement; U-LoA, Upper Limit of Agreement. *In the Bland-Altman method, the Mean Difference refers to the average of the difference in values obtained with the two methods, representing the accuracy of any given method in comparison to another method. The Limits of Agreement (LoA) refers to the limits within which $95 \%$ of all the scores fall on either side of the Mean Difference. This describes the precision of any tested method.

The results indicate a negative mean difference from the REs method for the attributes Order, Complexity, and Coherence in the black room and for the attribute Order in the white room. This means that REs measured at slightly lower ratings than the SIs for these attributes. For the rest of the attributes, results indicate that there was a positive mean difference from the RE (i.e., this method measured slightly higher ratings than the SIs). None of the attributes obtained a mean difference higher than the a priori criterion of $<1$, suggesting preliminarily that SI presentation is an accurate method to study the attributes presented in this study.

Yet, in the dataset of the attributes Spaciousness and Spatial Definition studied in the BR, the 95\% Confidence Interval (CI) for their mean difference is $0.32-1.09$ and $0.33-1.23$, respectively. Thus, there is a $95 \%$ probability that the true mean difference of the population is contained in the obtained CIs. These value ranges are higher than the a priori criterion of $<1$, meaning that the SI presentation cannot be used to evaluate these two attributes in rooms with black surfaces.

Thus, regarding the accuracy of SI as a method for daylighting studies, results suggest that SI presentation is an accurate method by which to evaluate the Pleasantness, Excitement, Order, Complexity, Legibility, Coherence, Spaciousness, Openness, and Spatial Definition of a small room with white surfaces and to evaluate the Pleasantness, Excitement, Order, Complexity, Legibility, Coherence, and Openness of a small room with black surfaces.

The precision of the method is related to the LoA. Results present considerable differences in the LoA, ranging from -4.35 to -1.88 for the lower LoA and from 1.55 to 4.70 for the upper LoA. Considering that the semantic differential scale used in this study includes seven steps divided among three 
(a)

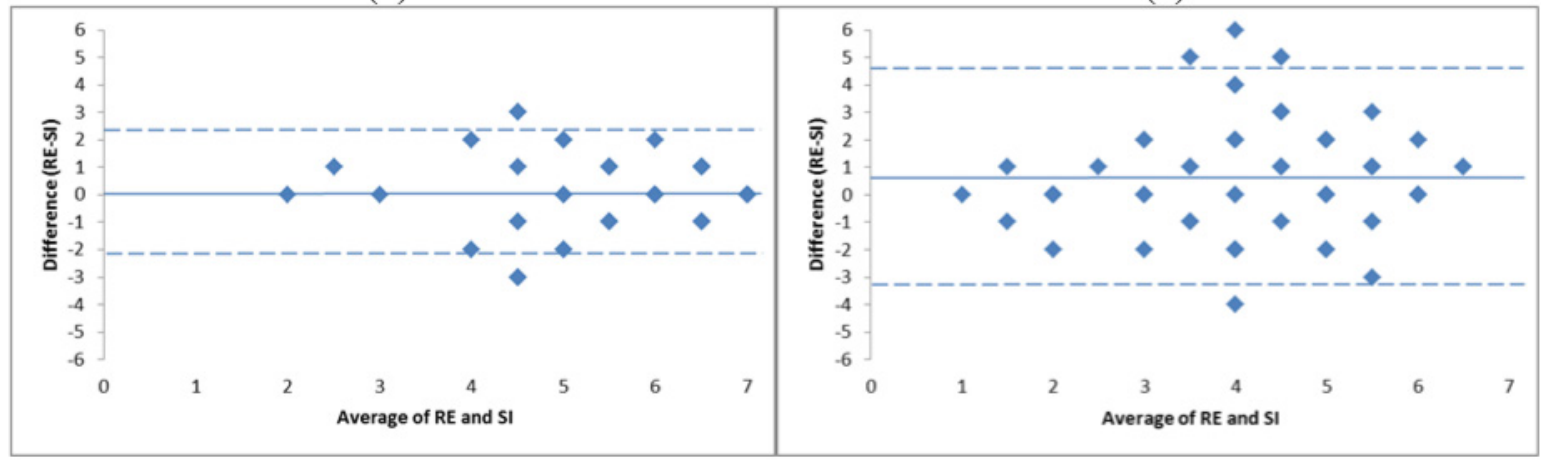

Fig. 4. Bland - Altman plots for spatial definition in (a) white room and (b) black room.

*Plot information: The X-axis represents the average of paired values from the Real Environment (RE) and Stereoscopic Imaging (SI) methods. The Y-axis represents the difference between the values of the RE and SI methods. The Mean Difference is expressed by the solid line, and the Limits of Agreement (both Upper and Lower) are represented by the dashed lines.

steps in one direction, three steps in the opposite direction, and one neutral step, the results indicate in nearly all cases a large difference in the LoA, thus suggesting lower precision of the SIs. The attribute Spatial Definition in the WR and the BR is presented graphically as example means (see Figure 4).

\section{DISCUSSION}

Before going deeper into the interpretation of the results, it seems important to reiterate that the purpose of this research was to study whether SI (under the described conditions and settings) could be used to study daylight and its impact on aesthetic perception and not whether SI can match the real luminance of a room. The automatic camera settings were used for taking all six sets of SI in order to negate dissimilar settings for different lighting conditions as an independent factor. By doing this, all nine selected attributes were evaluated under similar SI conditions. Thus, camera calibration for adopting similar luminance levels from REs was not contained in the scope of the study. The authors encourage further research on the calibration of equipment to match real luminances and how this affects daylighting studies. Other stereoscopic viewer technology, such as active technology or other types of passive technology, could also be a part of further research.

Nevertheless, it is necessary to comment on other technical practicalities that may have an impact on the results. Due to the parallel optical axes of the cameras, the loss of wide aspect ratio was a possibility. However, this seemed not to have a significant effect on participants' evaluations. This matter was specifically discussed during the debriefing interview. Most of the interviewed participants indicated that despite having an evident perceptual difference between SI and RE presentation, this did not have a large significance in their evaluations of the attributes. Their comments were later confirmed by the statistical analyses.

Despite the freedom given to the participants to choose their place from which to evaluate the SI, it was noted by the experimenter that no participant was walking during the evaluation. All participants were seated during their SI assessments. During the debriefing interview, only one of the participants indicated that it made more sense to sit on the right side of the laboratory when observing the viewpoint from the entrance of the room and on the left side when observing from the opposite viewpoint but that this did not affect the rooms' evaluations. Additionally, given that both SI and RE evaluations were controlled so that evaluations were made while facing the window wall and two sets of SI with 
different viewpoints were presented, it was ensured that the proportions of the projected scenes were perceived as similar to those in the REs.

Regarding numerical outcomes, it was anticipated that SI presentation could produce results similar to those from REs when performing the visual evaluation of a small room. According to the statistical analyses and findings, the experimental work presented in this article suggests that the validation of SI presentation as a research tool is supported for all studied architectural attributes in rooms with high luminances and for seven of the nine attributes in rooms with lower luminances. For the attributes Spaciousness and Spatial Definition in the BR, there is little statistical support to validate SI as a research method.

It was also expected that the WR would be a more suitable setting to be studied with SI than the BR. The assumption was that SIs with higher luminances could provide a significant increase in realism, making them amenable to be visually assessed in SI and obtaining more certainty in the scores. Additionally, later analyses of the participants' responses during the voluntary debriefing confirmed the numerical results. There seemed to be a response agreement that the WR was easier to assess with the SI than was the BR, especially on the attribute Spatial Definition. The results discussed in the previous paragraph and the lower values in the standard deviations from the WR compared to the BR seem to support this hypothesis.

These results answer the initial experimental question and suggest that, for the nine attributes studied, SI presentation may be used as an accurate experimental method when evaluating a small daylit room with only slightly lower or higher ratings than in an RE.

Nonetheless, the precision of SI should be discussed in comparison with the precision of the RE as the reference method for this study. The ratings obtained by the RE should hold a high precision value in order to compare it with the precision of the SI, but this is rarely possible in human and social research. The Bland-Altman approach is a statistical method, commonly used in medicine, where measurements are mostly independent from people's control. In an RE, where people control and decide their ratings, there is a higher risk of imprecision. Hence, the precision of the SI cannot be expected to achieve the precision that REs themselves do not possess. Furthermore, due to the design of the semantic differential scales, in which the positive poles are one side and the negative poles are on the opposite side, responses could interfere with measurement precision. This can be produced by response bias, either contraction bias, where participants avoid the extreme ends, or centering bias, where participants tend to rank evaluations in the middle of the scale [Zieliński et al. 2008].

However, the precision of the SI seems not to affect the findings about the accuracy of the method. It is important to remember that the sample size was increased from 26 to 78 observations (26 participants evaluated 3 different stimuli in each room for a replicated measurements analysis). Increasing the sample size would increase the precision but not the accuracy. Thus, we can be certain that the accuracy of the method has been proved and that SI can be used to study the listed attributes under certain conditions.

\section{CONCLUSION}

This study proposes SI as an alternative experimental method to the full-scale modeling of interiors in scientific experiments focusing on the impact of daylighting on the visual quality evaluation of architectural space. The study demonstrates that this tool can be used in a range of luminous scenes for the evaluation of certain architectural quality attributes. This can be important in the first phase of architectural and lighting design, when there is a need to evaluate large sets of different daylit configurations in small indoor spaces. The capacity of SI for reaching larger audiences of participants and shortening the experimental time can also be of important significance in many studies. 


\section{ACKNOWLEDGMENTS}

The authors gratefully acknowledge the contribution of Kine Angelo and Veronika Zaikina, members of the Light \& Colour Group of NTNU, for their assistance in conducting the experiment.

\section{REFERENCES}

Caitlin Akai. 2007. Depth Perception in Real and Virtual Environments: An Exploration of Individual Differences. Master's thesis, Simon Frasier University, Burnaby, BC, Canada.

Douglas G. Altman and J. Martin Bland. 1983. Measurement in medicine: The analysis of method comparison studies. The Statistician 32, 307-317.

Monica Billger, Ilona Heldal, Beata Stahre, and Kristian Renström. 2004. Perception of colour and space in virtual reality: A comparison between a real room and virtual reality models. In Proceedings of the IS\&T/SPIE 16th Annual Conference on Electronic Imaging, Vol. 5292. 90-98.

J. Martin Bland and Douglas J. Altman. 1986. Statistical methods for assessing agreement between two methods of clinical measurement. Lancet i, 307-310.

J. Martin Bland and Douglas J. Altman. 2007. Agreement between methods of measurement with multiple observations per individual. Journal of Biopharmaceutical Statistics 17, 571-582.

Yvonne de Kort, Wijnand Ijsselsteijn, Jolien Kooijman, and Yvon Schuurmans. 2003. Virtual laboratories: Comparability of real and virtual environments for environmental psychology. Presence 12, 360-373.

Marc Fontoynont, Dominique Dumortier, and Bruno Coutelier. 2007. Correlation of lighting quality descriptors with semantic characterization of luminous scenes. In Proceedings of the 26th Session of the CIE. D3-73-D3-76.

Michael Geuss, David Lessard, Jeanine Stefanucci, Sarah Creem-Regehr, and William Thompson. 2012. A comparison of size perception in real and virtual environments using judgments of action capability. Journal of Vision, 12, 9, article 912.

Stephen Kaplan and Rachel Kaplan. 1989. The visual environment: Public participation in design and planning. Journal of Social Issues 45, 59-86.

Ardeshir Mahdavi and Hesham Eissa. 2002. Subjective evaluation of architectural lighting via computationally rendered images. Journal of the Illuminating Engineering Society 31, 123-136.

Barbara Matusiak and Birgit Sudbø. 2008. Width or height? Which has the strongest impact on the size impression of rooms? Results from full-scale studies and computer simulations. Architectural Science Review 51, 165-172.

Claudia Moscoso and Barbara Matusiak. 2013. Virtual environment as a tool for daylighting studies. In Lux Europa: Proceedings of the 12th European Lighting Conference, Kraków, Poland.

Oi Naoyuki. 2005. The difference among generations in evaluating interior lighting environment. Journal of Physiological Anthropology and Applied Human Science 24, 87-91.

Jack L. Nasar. 2000. The evaluative image of places. In Person-Environment Psychology. New Directions and Perspectives (2nd ed.), W. Bruce Walsh, Kenneth H. Craik, and Richard H. Price (Eds.), Lawrence Erlbaum Associates, 117-168.

Guy R. Newsham, Roger G. Marchand, and Jennifer A. Veitch. 2002. Preferred surface luminances in offices, by evolution: A pilot study. In Proceedings of the IESNA Annual Conference.

Guy R. Newsham, C. Richardson, Catherine Blanchet, and Jennifer A. Veitch. 2005. Lighting quality research using rendered images of offices. Lighting Research and Technology 37, 93-112.

Masashi Okubo. 2011. Shape evaluation properties in real space and virtual space. In Emotional Engineering, Shuichi Fukuda (Ed.), Springer, 247-261. DOI: 10.1007/978-1-84996-423-4_13

Charles E. Osgood, George J. Suci, and Percy Tannembaum. 1971. The Measurement of Meaning. University of Illinois Press.

Anna Pellegrino. 1999. Assessment of artificial lighting parameters in a visual comfort perspective. Lighting Research and Technology 31, 107-115.

SIS-Swedish Standard Institute. 1998. NCS-färgprover - Betraknings - och mätvillkor samt toleranser (International Title: NCS-colour samples - Observation and measurement conditions and tolerances). STD-27101. Sweden: SIS/TK 433.

Iris Sprow, Zofia Barańczuk, Tobias Stamm, and Peter Zolliker. 2009. Web-based psychometric evaluation of image quality. In Proceedings of Image Quality and System Performance VI.

Arthur E. Stamps III. 1990. Use of photographs to simulate environments: A meta-analysis. Perceptual and Motor Skills 71, 907-913.

Arne Valberg. 2005. Light, Vision, Color. John Wiley \& Sons.

Celine Villa and Raphaël Labayrade. 2013. Validation of an online protocol for assessing the luminous environment. Lighting Research and Technology 45, 401-420. 
Jan Wienold, K. Beckinger, P. Apian-Bennewitz, and Cristoph Reinhart. 1998. Stationary Virtual Reality (SVR) - A new method for predicting user acceptance of daylighting systems. In Proceedings of the CIE Symposium on Lighting Quality, Ottawa, Canada.

Sławomir Zieliński, Francis Rumsey, and Søren Bech. 2008. On some biases encountered in modern audio quality listening tests - A Review. Journal of the Audio Engineering Society 56, 427-451.

Christine J. Ziemer, Jodie M. Plumert, James F. Cremer, and Joseph K. Kearney. 2009. Estimating distance in real and virtual environments: Does order make a difference? Attention, Perception, \& Psychophysics 71, 5, 1095-1106.

Received April 2014; revised June 2014, August 2014; accepted August 2014 